\title{
Defining System Changeability: Reconciling Flexibility, Adaptability, Scalability, and Robustness for Maintaining System Lifecycle Value
}

\author{
Adam M. Ross, Donna H. Rhodes, and Daniel E. Hastings \\ Massachusetts Institute of Technology \\ Building NE20-388, 77 Massachusetts Avenue \\ Cambridge, MA 02139 \\ adamross@mit.edu
}

Copyright $(2007$ by Adam Ross. Published and used by INCOSE with permission.

\begin{abstract}
Designing and maintaining systems in a dynamic contemporary environment requires a rethinking of how systems provide value to stakeholders over time. Classically, two different approaches to promoting value sustainment may include developing either alterable or robust systems. The first accomplishes value delivery through altering the system to meet new needs, while the second accomplishes value delivery through maintaining a system to meet needs in spite of changes. The definitions of flexibility, adaptability, scalability, and robustness are shown to be different parts of the core concept of "changeability," which can be described by three aspects: change agents, change effects, and change mechanisms. Cast in terms of system parameter changes, flexibility and adaptability are shown to relate to the origin of the change agent (external or internal to a system boundary respectively). Scalability and robustness, along with the additional property of modifiability, are shown to relate to change effects. The extent of changeability is determined by the number of possible change mechanisms available to the system as accepted by decision makers. Creating changeable systems, which can incorporate both classical notions of alterability and robustness, empowers systems to maintain value delivery over their lifecycle, in spite of changes in their contexts, thereby achieving value robustness to stakeholders over time.
\end{abstract}

\section{Introduction}

The need for "robust” systems. A U.S. Air Force/MIT Lean Aerospace Initiative Workshop on System Engineering for Robustness in June 2004 challenged the aerospace community to develop a process that enables systems engineering for "robustness" (Rhodes 2004). "Robustness" according to Dr. Marvin Sambur, Assistant Secretary of the Air Force for Acquisition at the time of the workshop, means:

- Capable of adapting to changes in mission and requirements;

- Expandable/scalable, and designed to accommodate growth in capability;

- Able to reliably function given changes in threats and environment;

- Effectively/affordably sustainable over their lifecycle;

- Developed using products designed for use in various platforms/systems; and

- Easily modified to leverage new technologies. 
These goals are similar to the separately defined ilities of adaptability, scalability, robustness, sustainability, and flexibility. Experts at the workshop admitted no comprehensive approach existed for designing for "robustness" in this sense and that further research was required in order to adequately address the Air Force need. One key problem raised was that the customer often wants to have ilities, but is not willing (or does not know how) to pay for them. A framework that allows for consideration of ilities during conceptual design, including concrete specification of how the ilities are defined and relate to perceived value, would provide value to the military and industry, and add clarity in understanding the value proposition for "robustness."

The desire for "robustness" stems from the fact that change is inevitable, both in reality and perception. Developing robust systems to deal with real-world changes has been advanced through such approaches as Axiomatic Design and Taguchi Robust Design methods (Suh 2001; Park 1996). The goal of system design is not robust systems per se, but rather the delivery of value to system stakeholders. Dealing with value mismatch, the difference between system offerings and stakeholder expectations, including dynamic changes, has not been dealt with in the same manner as classical notions of robustness. It is inevitable that decision makers change their mind, and therefore their perception of value of a system. In order to maintain value delivery over a system lifecycle, a system must change to match new decision maker preferences.

The motivation for changeability over a system lifecycle is categorized into three major drivers according to (Fricke and Schulz 2005): 1) dynamic marketplace, 2) technological evolution, and 3) variety of environments. These drivers result in two key aspects for system architectures to address: 1) they must be able to be changed easily and rapidly, and 2) they must be insensitive or adaptable towards changing environments. Such notions are often referenced in discussions of "flexibility" and "adaptability" and are often found in the manufacturing literature (Giachetti, Martinez et al. 2003). Recent work has tried to synthesize these definitions into a prescriptive six-element framework in the space system domain (Nilchiani 2005). Instead of focusing on individual "ilities," (Ross and Hastings 2006) introduce definitions for flexibility and adaptability as aspects of changeability and whose definitions are derived from both the technical literature and from common figurative usage, demonstrating their application in several aerospace case studies.

\section{Changeability Defined}

Change can be defined as the transition over time of a system to an altered state. If a system remains the same at time $\mathrm{i}$ and time $\mathrm{i}+1$, then it has not changed. The inevitability of the effects of time on systems and environments results in a constant stream of change, both of the system itself and of its environment.

A change event has three aspects: the agent of change, the mechanism of change, and the effect of change. Figure 1 displays these three aspects. The agent of change is the instigator, or force, for the change. The role of change agent can be intentional or implied, but always requires the ability to set a change in motion. The mechanism of change describes the path taken in order to reach state 2 from state 1 , including any costs, both time and money, incurred. The effect of change is the actual difference between the origin and destination states. A system that is black in time period one and gray in time period two has had its color changed. The change agent could be Nature, which can impart physical erosion due to wind, water, or sun, or could be a person with a paint can and brush. The change mechanism could be the erosion or painting process, costing no money, but taking a long time, or costing some amount of money, but taking 
a shorter amount of time. Figure 1 shows the three aspects of change that must be defined to specify a change.

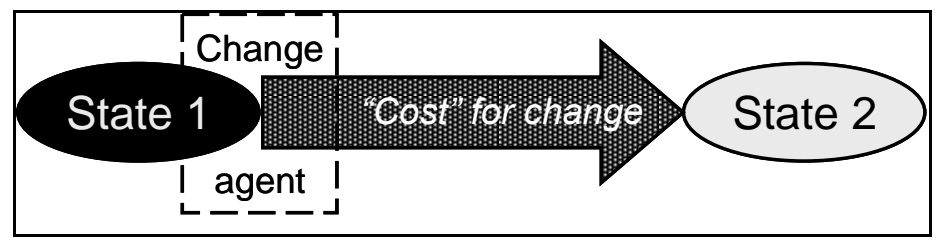

Figure 1. Change defined as state transition. Change specifications must include descriptions of change effect (difference between state 1 and state 2), change mechanism including "cost," and the change agent instigating the change.

Agents. A first aspect of change to define is change agent. As mentioned above, the agent is the force instigator for the change to occur. Examples of agents include humans, software, Mother Nature, etc. Intent is not required for a change agent, but the ability to set in motion a change mechanism is required. When characterizing an agent it is useful to think in terms of the steps required to put into motion an action. Working backwards from most to least necessary, three steps can be derived: 1) influence, 2) decision making, and 3) observation. Influence is the actual ability to set into motion a change mechanism. A physical impetus, such as a bat hitting a ball, or a social impetus, such as a boss ordering an employee, are examples of influence. Decisionmaking is the ability to process information in a structured manner in order to determine a course of action, presumably regarding whether to exert influence. Decision-making ability differentiates intentional actions from unintentional ones. Observation is the ability to gather relevant information in order to conduct effective decision-making. Observation can increase the likelihood of making good decisions. It is important to realize that action can occur with only influence present, but better action usually occurs with decision-making and the best action usually occurs with all three abilities present. Adding "intelligence" to a change agent is adding the abilities beyond influence. These three abilities are loosely based on the analysis in (Hall 1985), which differentiates between human and machine capabilities Psychomotor (Influence), Intellectual (Decision making), and Sensory/Perceptual (Observation).

The location of a change agent is a useful taxonomic distinction to be made when classifying change. If the change agent is external to the system, then the change under consideration is a flexible-type change. If the change agent is internal to the system, then the change under consideration is an adaptable-type change. If no change agent exists, then the system is rigid (no change can occur). Note that depending on the particular change being considered, a single system can be all three: flexible, adaptable, and rigid. Figure 2 summarizes these three change classification types.

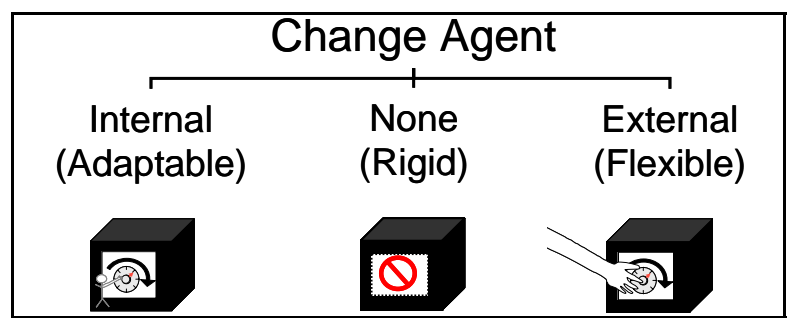

Figure 2. Change agent location for distinguishing between Adaptability, Rigidity, and Flexibility. 
Effects. A second aspect of change to define is change effect. The change effect is the difference in states before and after a change has taken place. Often it is the effect that is first noticed to indicate a change has occurred. A system can be described in terms of sets of parameters, which capture physical and functional aspects. Robustness is the ability to remain insensitive to change in parameters in spite of external changes. Scalability is the ability to change the level of a parameter. Modifiability is the ability to change the membership of the parameter set. As an example, consider the following parameter set, which includes both function and form: \{number of wheels, color of vehicle, quietness of cabin\}. Suppose a design under consideration has the following particular parameter values: $\{4$, "red," and "moderately quiet" $\}$. The possible ranges for these parameters include: $\{[3,4,6,8]$, ["black", "red”, "blue”], ["very quiet”, "moderately quiet", "little quiet", "not quiet"]\}. If the current system can maintain its \{four, "red," and "moderately quiet" $\}$ in spite of its operating environment changing, such as due to driving on unpaved roads, and past construction sites, then it is robust in these parameters to those particular environments. The more environments to which it is insensitive, the more robust the system.

If the system changes from having 4 to having 6 wheels, then it has made a scalable change, likewise if it changes from being "moderately quiet" to being "very quiet." If the system changes its parameterization set to be number of wheels, color of vehicle, quietness of cabin, fraction cabin open then it has undergone a modifiable change, enabling it to display a new function and/or form. For this example, the system is now designed to consider the amount of openness in the cabin. Figure 3 summarizes the three change effect types.

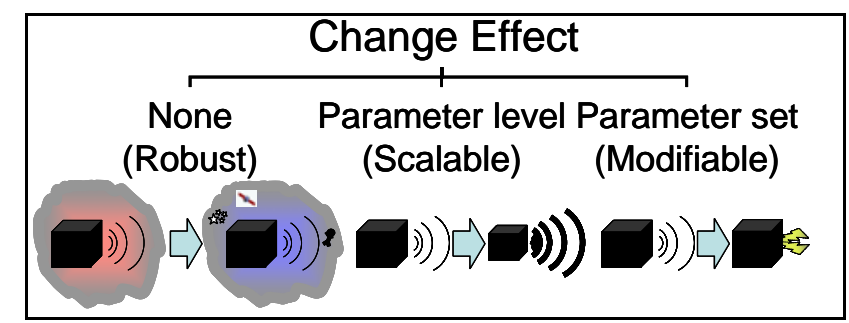

Figure 3. Change effect for classifying between Robustness, Scalability, and Modifiability.

Mechanisms. A third aspect of change to define is change mechanism. The mechanism is the path the system must take in order to transition from its prior to its post state. A change path details the necessary components to bring about the change, including conditions, resources, and constraints for the change. As an example, consider the problem of bringing a basketball down court from one basket to the other by a player. The change agent is the player, the system is the ball, and the change effect is the location of the ball. Many possible paths can be taken by the player, each incurring a "cost" in terms of time and effort to change the location state of the ball. Depending on the circumstances (e.g. location of opposing and supporting teams, time of the game, penalty status of the player) the player may choose different paths in order to bring about the same change. More generally, systems likewise have many potential change mechanisms for bringing about different change effects. The more change paths available for a system to follow, the more changeable the system. Just as the "cost" for following a path played a role in determining the best course of action for the basketball player, so too does the potential path cost for a system determine its apparent changeability for a given decision maker. The existence of paths may be objectively determined, but assessing whether a path is reasonable to follow is subjectively decided by individuals based on their preferences and circumstances, including 
available resources. Figure 4 gives a notional example multiple paths with differing costs connecting the same prior and post states for change.

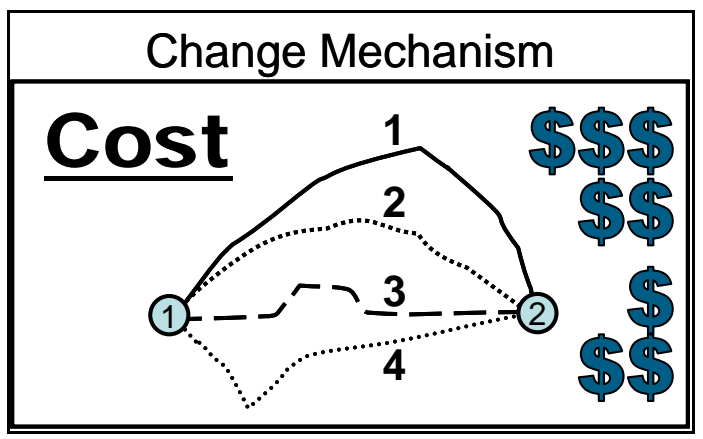

Figure 4. Change mechanism as paths for change for determining degree of changeability of a system.

Example. A designer wishes to determine the changeability of a computer system. If external change agents, such as users and technicians could make alterations to the system, then those alterations would be flexible-type changes. If internal change agents, such as automatic software updating, could make alterations to the system, then those alterations would be adaptable-type changes. In this way a computer is both flexible and adaptable, depending on the change under consideration. If the computer can have some of its parameters changed in level, then it is scalable, such as in memory. New capabilities can be added, to an extent, such as DVD-burners or TV-tuners, so the computer is modifiable in those new capabilities. Of course if the cost of any of these changes is too much for a particular user, then those changes would not be considered to be acceptable and would not count toward the changeability of the computer. The more low cost change mechanisms, both in terms of effort and dollars, the more changeable the computer will be perceived by the general user-base.

\section{Changeability Quantified}

Tradespace parameterization of systems. Quantification is a necessary next step in order to develop a concrete specification for changeability. A reasonable approach to comparing a large number of systems simultaneously is through a tradespace (Ross and Hastings 2005). Figure 5 below depicts the elements that go into tradespace development. Typically during concept exploration, a number of system designs and concepts are considered and assessed in terms of cost and benefit (i.e., value) to decision makers. The design parameter set, $\left\{D V^{N}\right\}$, represents the physical degrees of freedom for the system and can be assessed in terms of cost to develop, $C$, through the mapping $f_{C}:\left\{D V^{N}\right\} \rightarrow C$. 


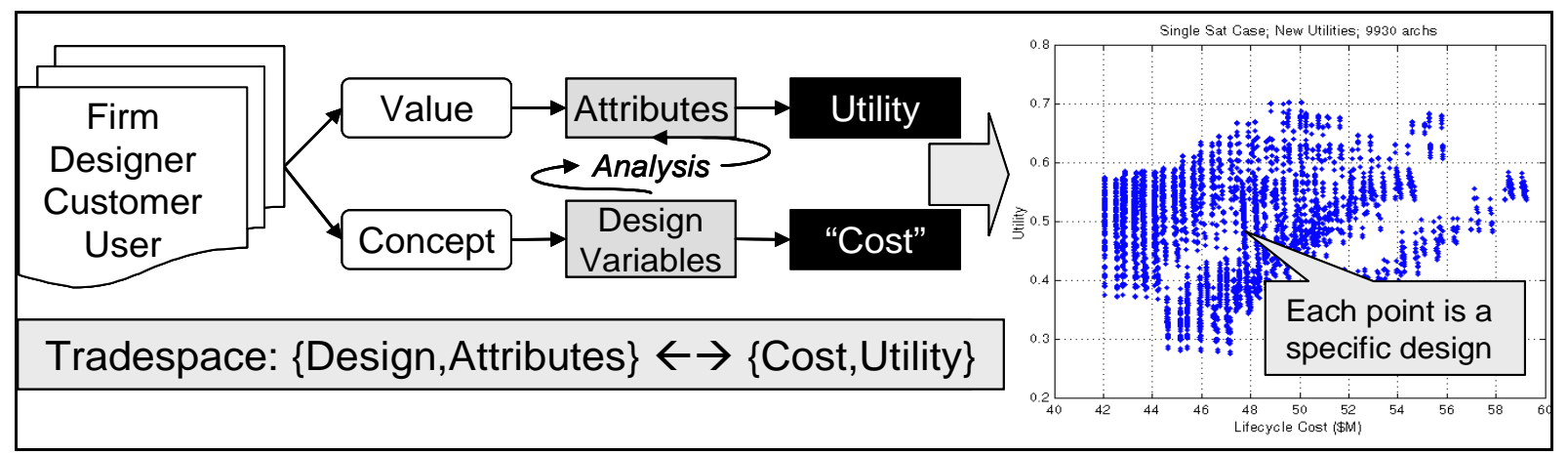

Figure 5.Tradespace defined as shorthand representations of designer controlled technical parameters and stakeholder perceived value parameters evaluated in terms of utility and cost (i.e., benefit and cost).

The attribute set, $\left\{X^{M}\right\}$, is a parameterization of value perceived by particular decision makers. Each decision maker specifies his or her own set with acceptable ranges, but whose specific values are derived from system designs being considered. The attributes can be aggregated in terms of value delivered to a decision maker through the concept of utility, $U$, with a function mapping $f_{U:}:\left\{X^{M}\right\} \rightarrow U$.

Tradespace networks: quantifying changeability through the Filtered Outdegree. The typical tradespace plot displays the system designs on a Cost-Utility space, showing the resources required (cost) and value delivered (utility) for the systems in a concise format. A Pareto Set characterizes those designs of highest utility at a given cost, across all costs. This set often shows the tradeoff of cost incurred for increased value. Considering each design as a potential starting or ending state for change, the tradespace frame suggests a mechanism for considering the changeability of system designs. As mentioned in the previous section, change specification requires a beginning state, an ending state, and a transition path. If in addition to specifying design parameters (static representations of a system) designers specify transition paths (dynamic change opportunities), a traditional tradespace can become a tradespace network.

A network is a model representation of nodes and arcs. Each node represents a location, or state, with each arc representing a path that connects particular nodes. In a tradespace network, system designs are nodes and the transition paths are arcs. The transition paths represent each of the potential change mechanisms available to a particular design. Figure 6 shows a traditional static utility-cost tradespace transformed into a tradespace network after the specification of transition rules, which are used to generate transition paths between design nodes. Transition rules, such as "burn on-board fuel to lower apogee" can be applied across a tradespace in an automated fashion to connect nodes efficiently. Designs that can follow more transition paths will have more outgoing arcs connecting it to other designs. In addition to representing an allowed transition, each arc has a cost associated with it, both in terms of dollars and time. 


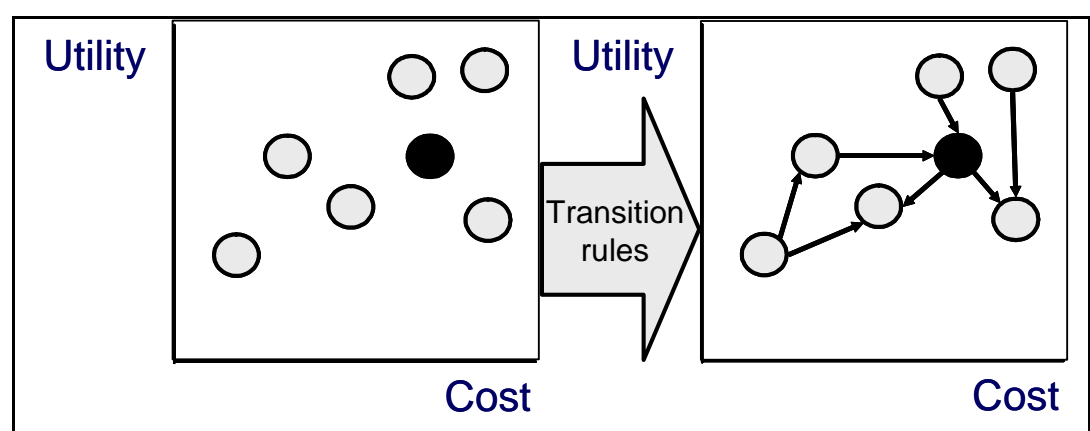

Figure 6. Specifying transition rules transforms a tradespace into a tradespace network, with transitionable designs accessible through heterogeneous arcs for each transition rule, or change mechanism.

Each decision maker will have an acceptability threshold for time or money spent for enacting change. The number of outgoing arcs from a particular design is called the outdegree for that design. The number of outgoing arcs from a particular design whose cost is less than the acceptability threshold, $\hat{C}$, is called the filtered outdegree for that design. The filtered outdegree is a quantification of changeability for a design for a decision maker. (See Figure 7 for a summary.) The higher the filtered outdegree of a design, the more changeable it is to that decision maker. The objective-subjective nature of the filtered outdegree captures the apparent relativity in perceived changeability of various designs: what may be flexible to one decision maker may not be flexible to another. The subjective acceptability threshold differentiates the results per decision maker. The objective outdegree calculation provides a mechanism for system designers to explicitly improve the potential changeability of a system: increase the number of outgoing arcs (add new transition rules), or reduce the cost of following outgoing arcs (increase the likelihood for arcs to cost less than acceptability threshold). Acceptability threshold "cost" can be on dollars, time, or any other resource that must be "spent" in order to follow a path.

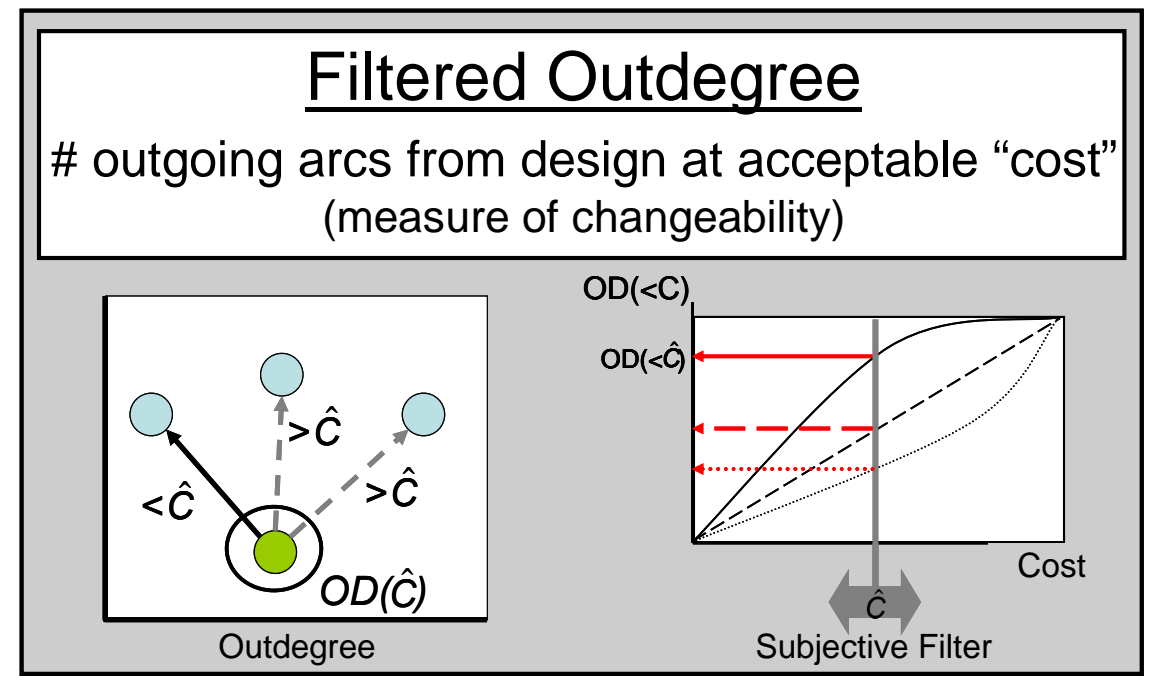

Figure 7. Filtered Outdegree, a measure of changeability, counts the number of potential transition paths available to a design, filtered by acceptable cost for change by a particular decision maker.

Other ilities quantified. Now that the more generalized changeability has been quantified, the question of quantifying the specific changeability types can be pursued. How does one determine 
if system A is more flexible than system B? Determine the filtered outdegree for systems A and B, only counting change mechanisms caused by system external change agents. For comparing adaptability, only count change mechanisms caused by system internal change agents. For quantifying in terms of the change effects, modifiability, scalability and robustness, formal equations can be defined.

Given a tradespace of size $S$, a vector mapping of design parameters to attributes $F_{X M}$, and a transition matrix $T_{i j k}$ keeping track of change mechanism costs for paths from design $i$ to design $j$ using mechanism $k$, the change effect ilities can be quantified per the following equations:

$$
\begin{array}{ll}
\text { modifiability }_{i}^{\mathrm{m}}: & \left.\sum_{j} \mid F_{X M}\left(D V_{i}\right) \cap F_{X M}\left(D V_{j}\right)=X^{m} \text { for } T_{i j k}<\hat{C}\right], \forall j, k \in S \\
\text { scalability }_{\mathrm{m}}^{\mathrm{m}}: & \left.\sum_{j} \mid\left[F_{X M}\left(D V_{j}\right)\right]^{m}-\left[F_{X M}\left(D V_{i}\right)\right]^{m} \neq 0 \text { for } T_{i j k}<\hat{C}\right], \forall j, k \in S \\
\text { value robustness } i \mathrm{ij}: & \left|U_{t 2}\left(F_{X M, t 2}\left(D V_{j}\right)\right)-U_{t 1}\left(F_{X M, t 1}\left(D V_{i}\right)\right)\right| \approx 0, \text { for }_{i j k}<\hat{C}, \forall j, k \in S
\end{array}
$$

For example, modifiability ${ }_{i}^{m}$, or the modifiability of system $i$ in attribute $m$, is the number of paths connecting $i$ to designs that are the same as $i$ except it has attribute $m$ added or subtracted from its attribute set, but whose transition mechanism costs less than the acceptability threshold.

Examples. The following are a few examples to illustrate the five changeability concepts introduced in this paper: flexibility, adaptability, scalability, modifiability, and value robustness.

Flexible Change. A debate often cited within MIT circles entails the apparent conflict between two professors' definitions of flexibility. On the one hand, Professor A believes that networks, such as a computer network, are highly flexible, while a space system is not. Professor B believes that a space system that can be changed from halfway across the solar system to be able to perform a new mission is flexible, where the comparison to the flexibility of a computer network is irrelevant. The definition for flexibility given above reconciles these two views and provides a good example for the definition.

The question of whether a system is flexible is the same as asking whether $\operatorname{cost}\left(D V_{i} \rightarrow D V_{j}\right)<$ $\left(\hat{C}, t^{\imath}\right)$, as accomplished by a system-external agent. For the computer network question, the physical change could correspond to physical network changes (wires=arcs), computers (nodes), or informational, such as routing of packets. For these classes of changes of a computer network, the cost to change can vary from thousands of dollars down to the order of pennies, or $\$ 10^{3}-10^{-2}$. Additionally, the time for a change can vary from hours down to fractions of a second, or $10^{3}-10^{-}$ ${ }^{2}$ min. For the space system in question, the physical change could correspond to the change of on-board payloads, or loaded operational software. For these classes of changes of a space system, the cost to change can vary from billions to hundreds of thousands of dollars, or $\$ 10^{9}$ $10^{5}$. Additionally, the time for a change can vary from years to days, or $10^{6}-10^{3} \mathrm{~min}$. The key point here is the subjectively set threshold for change acceptability, $\hat{C}, t^{\hat{n}}$, cost and time respectively. For Professor A, these thresholds are probably on the order of $10^{-1}$ and $10^{-1}$, respectively, while for Professor $B$, these are probably on the order of $10^{6}$ and $10^{5}$, respectively. In a sense, both professors are correct and consistent in their definitions. An important distinction to make, however, is that if the change agent for these two cases is located inside the system boundary, the change is considered an adaptable one. For this example we consider outside 
human intervention as the cause for change, though one could imagine a self-changing network or autonomous satellite, especially if the time scales for change are beyond the capabilities of a human (See (Hall 1985) for examples of time scale and spatial scale comparison of human and machine task abilities and limitations.)

Adaptable Change. As highly adaptable systems, humans constantly employ their Psychomotor, Intellectual, and Sensory/Perceptual abilities to create change of themselves. Consider weighttraining and other exercise as a long term change from an "out-of-shape" system to a "fit-as-afiddle" system. Those people (systems) who perceive the cost of doing such an adaptable change as acceptable, execute on it, i.e, the question of whether to pursue an adaptable change through the exercise mechanism is whether $\operatorname{cost}\left(D V_{i} \rightarrow D V_{j}\right)<?\left(\hat{C}, t^{n}\right)$, as accomplished by a systeminternal agent (the self). In other "words": (cost $\left(D V_{\text {outofshape }} \rightarrow D V_{\text {fitasfiddle }}\right)<$ ? (max_willingness_to_pay_gym/trainer, max_willingness_to_wait_for_results).

The general consensus that humans are highly adaptable is because over a very large range of possible and actual changes, people decide that the cost is acceptable (i.e. the cost of self-change is reasonable, both in terms of dollars and time). Making a person more adaptable entails either decreasing the actual cost for the change, or increasing the cost threshold for the change. Personal training can have either effect (by increasing the number of mechanisms for personal growth on the one hand, and by increasing a person's patience on the other hand).

Scalable Change. Suppose a consumer has a camera and cares about its megapixel rating, $X^{\text {Megapixel }}$. The current rating for the camera is $X_{\text {Megapixel }}{ }_{\text {current }}=4.0$. He now desires that the camera have $X^{\text {Megapixel }}$ future $=7.0$. The change in a level of a current attribute, megapixel rating, is an example of a scalable change. The question facing the consumer is whether $\operatorname{cost}\left(X^{M}\right.$ current $\left.\rightarrow X^{M}{ }_{\text {future }}\right)<$ ? $\left(\hat{C}, t^{*}\right)$. The mechanism for achieving the change must be determined through the inverse mapping $F_{X M}{ }^{-1}, F_{X M}^{-1}:\left\{X^{M}\right\} \rightarrow\left\{D V^{N}\right\}$, for each attribute current and future. What design parameters need to change and what does it cost in order to effect a scalable change in megapixels? Possible answers include modification of the optics and charge-coupled device (CCD) photon receiver in the current camera, or throwing out the current camera and purchasing a new one (depending on the consumer, the costs for these mechanisms may vary, as will the subjective cost threshold... a do-it-yourself engineer may prefer the modification route, while the typical user may prefer the new purchase). In any case, if the cost for change is acceptable, the camera system has undergone a scalable change for the megapixel attribute.

Modifiable Change. A simple example of a modifiable change is that which is often done to a personal computer. Suppose a decision maker has a standard computer with a monitor, keyboard, mouse, and hard drive, all of the standard features of a computer purchased circa 1999. Changes occur in the market place, with new technologies being offered to enhance computer capabilities. The decision maker decides at some point later in time that he really would like to be able to burn DVDs. That capability was absent from the original computer system, but with a simple change to the computer, that capability can be added. The decision problem posed to the decision maker is whether $\operatorname{cost}\left(\left\{X^{M}\right\} \rightarrow\left\{X^{M}\right\} \cup\left\{X^{D V D b u r n i n g}\right\}\right)<$ ? $\hat{C}$. In order to determine the answer, the inverse mapping $F_{X M}{ }^{-1}, F_{X M}^{-1}:\left\{X^{M}\right\} \rightarrow\left\{D V^{N}\right\}$, must be determined. Luckily the design problem has already been solved through modular design. All that is needed is the addition of an external (or internal) DVD-R drive. In this case, the mapping between design- and value-space is straightforward, $X^{\text {DVDburning }} \rightarrow D V^{\text {DVDburner. }}$. Thus, is the addition of the $D V^{\text {DVDburner }}<\hat{C}$ ? (Of course, this is the question posed by most consumers when shopping for computer additions.) The beauty of modular design is that the cost of additional modules is often relatively low, 
thereby increasing the likelihood that the cost for change is less than $\hat{C}$.

Value Robustness. Suppose a decision maker looking to procure a new box has two attributes: size and loudness. Four system offerings exist and are given in Figure 8 below. Size is more important than loudness. Within size, big is preferred to small. Within loudness, loud is preferred to quiet. Applying these preferences, the systems can be ordered according to their utility:

$$
\mathrm{U}(3)>\mathrm{U}(2)>\mathrm{U}(1)>\mathrm{U}(4)
$$

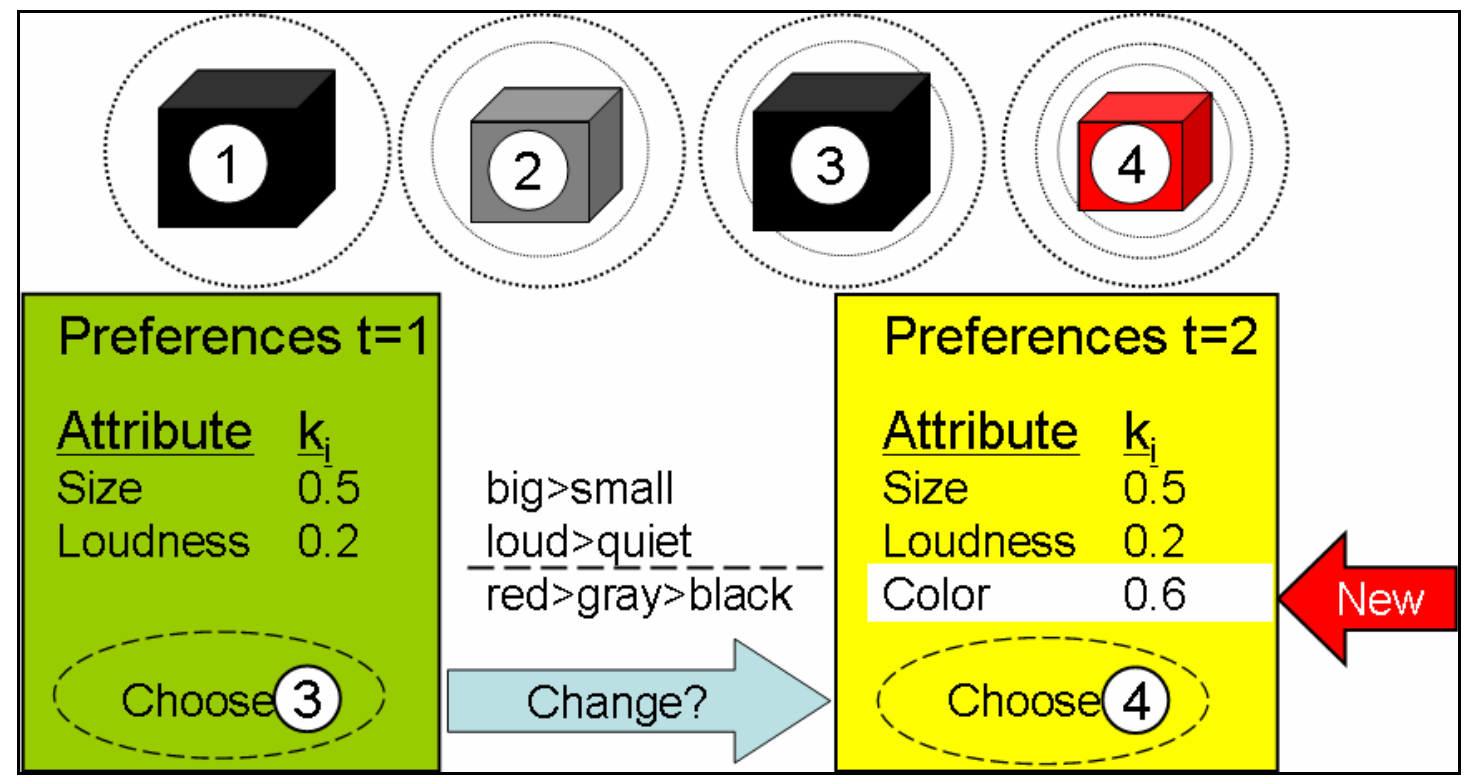

Figure 8 Example value robustness: Choosing boxes

Thus the decision maker should choose system offering (3) in order to maximize his value. Suppose something happens and the decision maker now cares about color as well as size and loudness. Color is about as important as size, which is more important than loudness. Within color, red is preferred to gray is preferred to black. Applying these new preferences, the systems can be ordered according to their utility at time $\mathrm{t}=2$ :

$$
\mathrm{U}(4)>\mathrm{U}(2)>\mathrm{U}(3)>\mathrm{U}(1)
$$

Notice that at time $t=2$ the decision maker should choose system (4) in order to maximize his value. If, however, the decision maker must choose a system at time $t=1$, the best choice is to choose system (3) and then change it to system (4) at time $t=2$. If the switching costs are high, however, the net value to the decision maker might be less than choosing system offering (2), which is second best in both time periods and entails no switching cost. Offering (2) is robust in value under this preference change. In this example, $U_{t 2} \neq U_{t 1}$, and value robustness can be achieved by having $D V_{i}=D V_{j}$ when switching costs are high, i.e., $\operatorname{cost}\left(D V_{3} \rightarrow D V_{4}\right)>\hat{C}$. Or can be achieved by having $D V_{1} \neq D V_{j}$, when switching costs are low enough, i.e., $\left(\operatorname{cost}\left(D V_{3} \rightarrow D V_{4}\right)<\right.$ $\hat{C}$. The first case suggests the decision maker choose $D V_{i}=D V_{j}=D V_{2}$, and the second case suggests the decision maker choose $D V_{i}=D V_{3}$, and $D V_{j}=D V_{4}$.

Choosing a passively value robust design requires finding designs that remain high value perceived across various future scenarios. The Pareto Set in a tradespace contains those designs 
that have highest utility at fixed cost, across all costs. Passive value robustness can be quantified as the Pareto Trace number, which is the number of Epochs whose Pareto Set contains that particular design, reflecting the designs that have the most efficient utility for a given level of resource expenditure. An Epoch is a time period that bounds a contextual scenario, during which stakeholder expectations, constraints, design concepts, available technologies, and policies are defined. Figure 9 shows the Pareto Trace number definition along with a tradespace over time plot showing the Pareto Trace designs that remain in the "optimal" set over the various Epochs. A relatively large Pareto Trace number implies a high passive value robustness factor for a particular design and can be a function of excess capability, insensitivity to the particular change scenario, or a locally stable region in a tradespace.

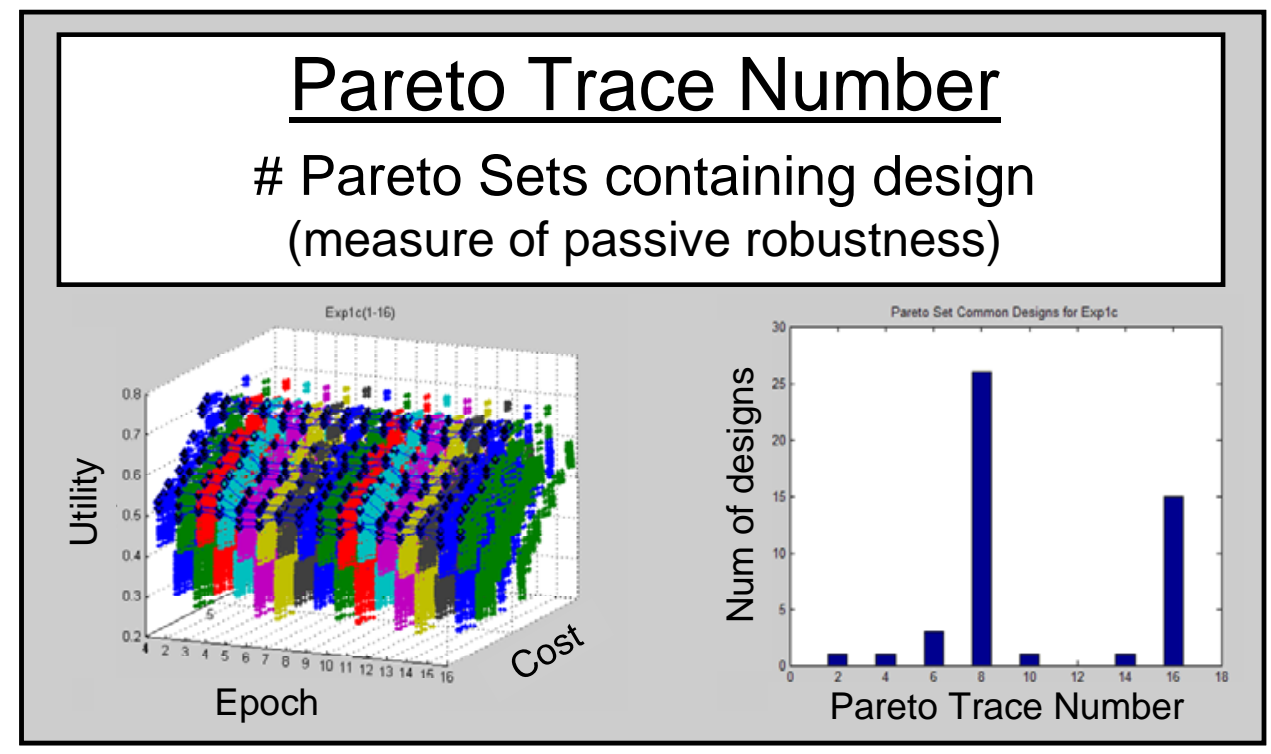

Figure 9. Passive value robustness as Pareto Trace Number.

\section{Discussion}

Relation to Valuation Methods. While the identification and quantification of the ilities was explicitly undertaken in this work, valuation of the ilities was intentionally excluded from consideration. Valuation of ilities is an additional layer of analysis that can be put on top of the proposed framework. The reason valuation was excluded is that all valuation techniques rely upon specific assumptions regarding how to collapse time, utility, and cost into a single metric. Layering such assumptions into the framework would obfuscate the interesting trades which exist among these three quantities. In effect, the ilities are being valued through the decision process itself.

As pointed out in (Roberts 2003), the ability to change the onboard delta- $v$ for the Spacebased Radar system represents a real option for the system. This capability can be described by the existence of a "path-enabling" variable, which is a parameter that reduces the cost or allows for particular change mechanisms to be followed. An example of a path enabling parameter is the use of "commercial-of-the-shelf" (COTS) parts, which reduces the cost of replacing or building components. Another example is the "amount of modularity used." (Ross 2006) gives examples of path enablers in case studies of the Terrestrial Planet Finder (TPF) astronomical system and the Joint Direct Attack Munition (JDAM) bomb modification kit systems. Path enabling 
variables differ from design variables in that design variables are generated in order to create value, while path enablers are generated in order to create or enhance changeability. Real options and path-enabling variables are similar in that both factors allow for a system change, and may not contribute to system value if left unused. Valuation of path-enabling variables can be done utilizing real options analysis, and likewise, identification of real option opportunities can benefit from the definition of path-enabling variables. The present work is considered to be both preceding and complementary to traditional real options analysis. The ongoing MIT work on defining real options "in" systems tends to focus more on valuation, rather than discovery of the key variables that should be investigated for real options. An exception is (Bartolomei, Hastings et al. 2006), whose research is trying to discover "hot spots" or key system aspects that should be addressed for leveraging changeability (flexibility), that is identifying real option opportunities.

Dealing with Uncertainty. One of the key motivations for system changeability is the inevitability of change in the future system context. Not knowing the specifics is akin to admitting uncertainty in future markets, technologies, and environments. Other types of uncertainty also exist, both regarding past and present factors affecting system value. Instead of motivating flexibility as a response to uncertainty, the present work is motivating changeability as a response to inevitable change, which is the true salient issue in the concern over uncertainty. Building in changeability into systems admits the inability to accurately predict the future, while embracing the Bayesian technique of constantly improving estimates given new information. No longer does a system have to settle for putting off fixing its requirements for as long as possible in order to better match "actual" preferences. A changeable system can change to meet the "actual" and future preferences of stakeholders given improved information revealed over time.

Tension between Changeability and Robustness. One common perception is that system properties are in tension, such as flexibility and robustness, where one must give up one to have more of the other. Such is not necessarily the case; it depends on the parameters under consideration. For example, making a computer robust to noise and physical impacts does not reduce its modifiability for changing components. The robustness of the system is embedded in its modular design; each module employs robust design principles and together the overall system maintains robustness. Robustness obtained through redundancy may suffer through modularization due to the reduction of interconnections inherent in modular design (See (Fricke and Schultz 2005) for discussion of the tensions between modularity and redundancy.) But modularization is just one approach towards achieving changeability through its ability to reduce the costs for change. Likewise, redundancy is just one approach towards achieving robustness.

At a higher level is the concept of value robustness. If the goal for system design and development is to deliver value to stakeholders over the system lifecycle, then value robustness is the ultimate goal for the designers. Value robustness can be achieved through either passive or active means, with the former more akin to traditional robust approaches, and the latter embracing changeability as a dynamic strategy for value sustainment. Passive value robustness delivers value through the development of "clever" designs that are perceived to maintain value over time. Successful development of passively value robust systems requires a bit of luck as well as anticipation of future system context, including potential value perceptions and the competitive environment with alternative systems. Active value robustness requires less omniscience, but does have the added complexity of needing a change agent changing the system over time to maintain high value perception.

Figure 10 depicts the two value robustness strategies. The teal circle design follows the passive value robust strategy shown by the dashed line, while the green cross design follows the 
active value robust strategy shown by the solid line. The top portion of the figure shows utility versus time across two time periods, while the bottom portion shows utility versus cost at the beginning of the first time period and the end of the second time period respectively. Comparing the utility-cost tradespaces show both value robust strategies result in "constant" utility across changing context, though the active strategy results in a different end design than the passive strategy.

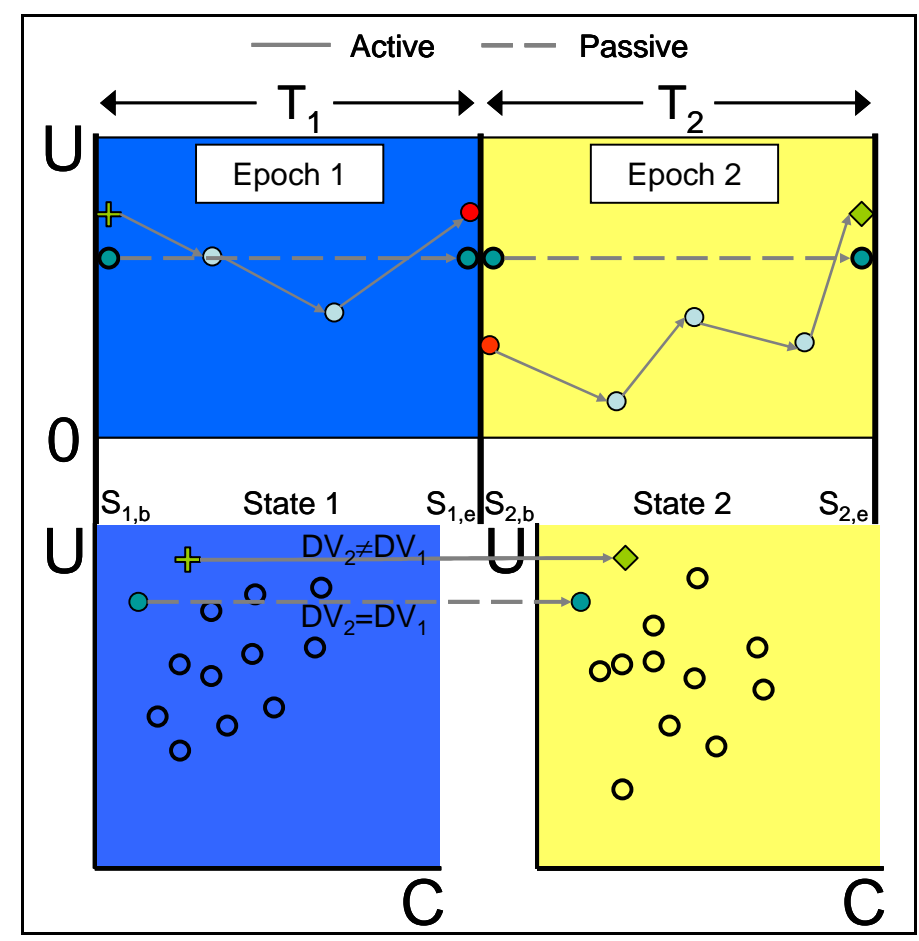

Figure 10. Active versus passive value robust strategies across two time periods.

Implications for Design. Given the motivation of a value-centric definition of changeability and robustness, the implications for design become clear. Designers must no longer only consider the aspects of a design that meet today's needs and requirements, but rather keep an eye to the future by building in change mechanisms into a system to allow for change. Designers can parameterize the system both in terms of design parameters as well as path enablers, the former intended to create value and the latter intended to lower the cost or create the possibility for following a change path. Creating intelligent systems or enterprises can be considered explicitly as the internalization of change agents. Guiding designers in addition to the concrete definitions in this paper are the heuristics and guiding principles in (Fricke and Schulz 2005) which lists three basic principles and six extending principles. These principles are techniques that will allow designers to creatively develop change mechanisms that will more likely be acceptable to decision makers.

Implications for Policy. One key drawback to adding changeability into systems is the potential for adding cost through unused change mechanisms. While not necessarily required, adding "real options" often has a carrying cost associated with it and if unused, adds to the overall cost with no perceived benefit, making them unattractive to decision makers with limited budgets. Acquisition policy must allow for the positive accounting for unused change mechanisms through allowing for the carrying of unused change costs by appropriate organizations. As an example, consider the development of the JDAM system, which was developed by the Boeing 
Company. Boeing was allowed to have class 2 change authority on the system, as well as maintain ownership over the design itself. These two policies empowered Boeing to add path enablers to the system that lowered the cost for future change, but may in the short term have added costs (Ross 2006).

\section{Conclusion}

The system "robustness" requested by Dr. Sambur is in fact value robustness, a strategy that may use various other ilities in its attempt to maintain system value delivery over time, in spite of context changes. Passive value robustness more closely approximates traditional notions of robustness through the pursuit of developing systems insensitive to external changes. Active value robustness embraces changeability and through dynamic matching of system offerings to stakeholder expectations given varying contexts, can provide value over time even in highly uncertain or unexpected future scenarios.

The changeability definitions discussed in this paper provide a concrete basis for conversation about the ilities, specification for design, analysis techniques for comparison, and a new paradigm for considering systems as dynamic constructs for creating value over time. Designing for value robustness is a sound dynamic strategy for maintaining system lifecycle value.

\section{Acknowledgements}

The author gratefully acknowledges the financial support for this research from the Lean Aerospace Initiative (LAI), a consortium including MIT, government, and industrial members of the aerospace industry, and intellectual support from the MIT Systems Engineering Advancement Research Initiative $\left(\mathrm{SEA}^{\mathrm{RI}}\right)$.

\section{References}

Bartolomei, J. E., D. E. Hastings, et al., "Screening for Real Options "In" an Engineering System: A Step Towards Flexible System Development--PART I: The Use of Design Matrices to Create an End-to-End Representation of a Complex Socio-Technical System”, INCOSE Conference on System Engineering Research, Los Angeles, CA, 2006.

Fricke, E. and Schulz, A.P., "Design for Changeability (DfC): Principles to Enable Changes in Systems Throughout Their Entire Lifecycle.” Systems Engineering 8(4), 2005.

Giachetti, R. E., L. D. Martinez, et al., "Analysis of the structural measures of flexibility using a measurement theoretical framework," International Journal of Production Economics 86: 47-62, 2003.

Hall, S. B., Ed., The Human Role in Space: Technology, Economics and Optimization, Noyes Publications, Park Ridge, NJ, 1985.

Nilchiani, R. "Measuring the Value of Space Systems Flexibility: A Comprehensive Six-element Framework". Ph.D. in Aeronautics and Astronautics, Massachusetts Institute of Technology, Cambridge, MA, 2005.

Park, S. H., Robust Design and Analysis for Quality Engineering, Chapman \& Hall, New York, 1996.

Rhodes, D.H., Air Force/LAI Workshop on Engineering for Robustness, Technical Report, Lean Aerospace Initiative, http : / / lean .mit . edu, July 2004.

Roberts, C.J., "Architecting Strategies Using Spiral Development for Space Based Radar", S.M. 
in Technology and Policy Program, Massachusetts Institute of Technology, Cambridge, MA, June 2003.

Ross, A.M., "Managing Unarticulated Value: Changeability in Multi-Attribute Tradespace Exploration.” Ph.D. in Engineering Systems, Massachusetts Institute of Technology, Cambridge, MA, June 2006.

Ross, A.M., and Hastings, D.E. “The Tradespace Exploration Paradigm”, INCOSE 2005 International Symposium, Rochester, NY, July 2005.

Ross, A.M., and Hastings, D.E., "Assessing Changeability in Aerospace Systems Architecting and Design Using Dynamic Multi-Attribute Tradespace Exploration.” AIAA Space 2006, San Jose, CA, September 2006.

Suh, N.P., Axiomatic Design--Advances and Applications. Oxford University Press, New York, 2001.

\section{Biography}

Adam M. Ross. Dr. Adam M. Ross is a Research Scientist in the MIT Engineering Systems Division. His research focuses on managing unarticulated value, designing for changeability, and dynamic tradespace exploration for complex systems. Dr. Ross received his Ph.D. from the MIT Engineering Systems Division in June 2006, and was previously a research assistant with the MIT Lean Aerospace Initiative. Dr. Ross has published papers in the area of space systems design. He has work experience with government, industry, and academia including NASA Goddard, JPL, Smithsonian Astrophysical Observatory, Boeing Satellite Systems, MIT, Harvard, and Florida State University, performing both science and engineering research.

Donna H. Rhodes. Dr. Donna H. Rhodes is a Senior Lecturer and Principal Research Scientist in the MIT Engineering Systems Division. Her areas of specialization include technical and management theory and practices for architecting and design of complex systems, systems-ofsystems, and enterprises. Prior to joining MIT, Dr. Rhodes had 20 years of experience in the aerospace, defense systems, systems integration, and commercial product industries. Dr. Rhodes is a Past President and Fellow of INCOSE, and is a recipient of the INCOSE Founders Award and several INCOSE Distinguished Service Awards. She has published numerous papers and research reports in the field of systems, and has co-authored industry and corporate engineering policies, standards, and technical reports.

Daniel E. Hastings. Dr. Daniel E. Hastings is a Professor of Engineering Systems and Aeronautics and Astronautics, and Dean for Undergraduate Education at MIT. Dr. Hastings' research concentrates on issues of space systems and space policy. He has published papers and a book on spacecraft-environment interactions and papers on space propulsion and space systems. Dr. Hastings is an AIAA Fellow and a member of the International Academy of Astronautics. He is serving as a member of the National Science Board, the Applied Physics Lab Science and Technology Advisory Panel, and as past chair of Air Force Scientific Advisory Board. He served as chief scientist to the U.S. Air Force, and has led several national studies on government investment in space technology. 